Algebraic $8 \mathcal{G}$ Geometric $\mathcal{T}$ opology

Volume 1 (2001) 427-434

Published: 31 July 2001

ATG

\title{
Maximal Thurston-Bennequin Number of Two-Bridge Links
}

\author{
LENHARD L. NG
}

\begin{abstract}
We compute the maximal Thurston-Bennequin number for a Legendrian two-bridge knot or oriented two-bridge link in standard contact $\mathbb{R}^{3}$, by showing that the upper bound given by the Kauffman polynomial is sharp. As an application, we present a table of maximal ThurstonBennequin numbers for prime knots with nine or fewer crossings.
\end{abstract}

AMS Classification 53D12; 57M15

Keywords Legendrian knot, two-bridge, Thurston-Bennequin number, Kauffman polynomial

\section{Introduction}

A Legendrian knot or link in standard contact $\mathbb{R}^{3}$ is a knot or link which is everywhere tangent to the two-plane distribution induced by the contact oneform $d z-y d x$. Given either a Legendrian knot or an oriented Legendrian link, we may define its Thurston-Bennequin number, abbreviated $t b$, which is a Legendrian isotopy invariant; see, e.g., [1] or [6]. (Henceforth, we will use the word "link" to denote either a knot or an oriented link.) For a fixed smooth link type $K$, the set of possible Thurston-Bennequin numbers of Legendrian links in $\mathbb{R}^{3}$ isotopic to $K$ is bounded above; it is then natural to try to compute the maximum $\overline{t b}(K)$ of $t b$ over all such links. Note that we distinguish between a link and its mirror; $\overline{t b}$ is often different for the two.

Bennequin [1] proved the first upper bound on $\overline{t b}(K)$, in terms of the (threeball) genus of $K$. Since then, other upper bounds have been found in terms of the HOMFLY and Kauffman polynomials of $K$. The strongest upper bound, in general, seems to be the Kauffman bound first discovered by Rudolph [11], with alternative proofs given by several authors; see [5] for a more detailed history of the subject. 
Let $F_{K}(a, x)$ be the Kauffman polynomial of a link $K$, and let min-deg ${ }_{a}$ denote the minimum degree in the framing variable $a$. With the normalizations of [6], the Kauffman bound states that

$$
\overline{t b}(K) \leq \min -\operatorname{deg}_{a} F_{K}(a, x)-1 .
$$

The Kauffman inequality is not sharp in general; see, e.g., [4, 5]. Sharpness has been established, however, for some small classes of knots, including positive knots [13], most torus knots [3, 4], and most three-stranded pretzel knots [9]. In this note, we will establish sharpness for a somewhat "larger" class of links, the 2-bridge (rational) links. (We remark that the HOMFLY bound is not sharp in general for this class.)

Theorem 1 If $K$ is a 2-bridge link, then $\overline{t b}(K)=\min _{-d_{a} g_{a}} F_{K}(a, x)-1$.

Theorem 1 will be proved in Section 2 .

Recall that a 2-bridge link is any nontrivial link which admits a diagram with four vertical tangencies (two on the left, two on the right). This class of links includes many prime knots with a small number of crossings. More precisely, all prime knots with seven or fewer crossings are 2-bridge, as are all prime knots with eight or nine crossings except the following: $8_{5}, 8_{10}, 8_{15}-8_{21}, 9_{16}, 9_{22}$, $9_{24}, 9_{25}, 9_{28}, 9_{29}, 9_{30}$, and $9_{32}-9_{49}$.

Hand-drawn examples by N. Yufa and the author [14] show that the Kauffman bound is sharp for all of the above non-2-bridge 8-crossing knots, except for $8_{19}$ (more precisely, the mirror image of the version drawn in [10]). Since 819 is the $(4,-3)$ torus knot, a result of $[4]$ yields $\overline{t b}=-12$ in this case, while the Kauffman bound gives $\overline{t b} \leq-11$. Inspection of the non-prime knots with eight or fewer crossings shows that the Kauffman bound is sharp for all such knots. We thus have the following result.

Theorem 2 The Kauffman bound is sharp for all knots with eight or fewer crossings, except the $(4,-3)$ torus knot 819 .

Further drawings show that the Kauffman bound is sharp for all of the 9crossing prime knots which are not 2 -bridge, except possibly for $9_{42}$ (more precisely, the mirror of the $9_{42}$ diagram in [10]). For this last knot, we believe that $\overline{t b}=-5$, while Kauffman gives $\overline{t b} \leq-3$.

An appendix to this note provides a table of $\overline{t b}$ for prime knots with nine or fewer crossings. Note that this table improves on the one from [13], which 
only considers one knot out of each mirror pair, and which does not achieve sharpness in a number of cases.

Acknowledgements The author would like to thank Nataliya Yufa for her careful drawings which culminated in Theorem 2 and the appendix table, Tom Mrowka and John Etnyre for useful discussions, and Isadore Singer for his encouragement and support. This work was partially supported by grants from the NSF and DOE.

\section{Proof of Theorem 1}

Let $K$ be a 2-bridge link; we first need to find a suitable Legendrian embedding of $K$. Say that a link diagram is in rational form if it is in the form $T\left(a_{1}, \ldots, a_{n}\right)$ illustrated by Figure 1 for some $a_{1}, \ldots, a_{n}$. Clearly any rational-form diagram corresponds to either the trivial knot or a 2-bridge link; by the classification of 2-bridge links [12], any 2-bridge link has a rational-form diagram.
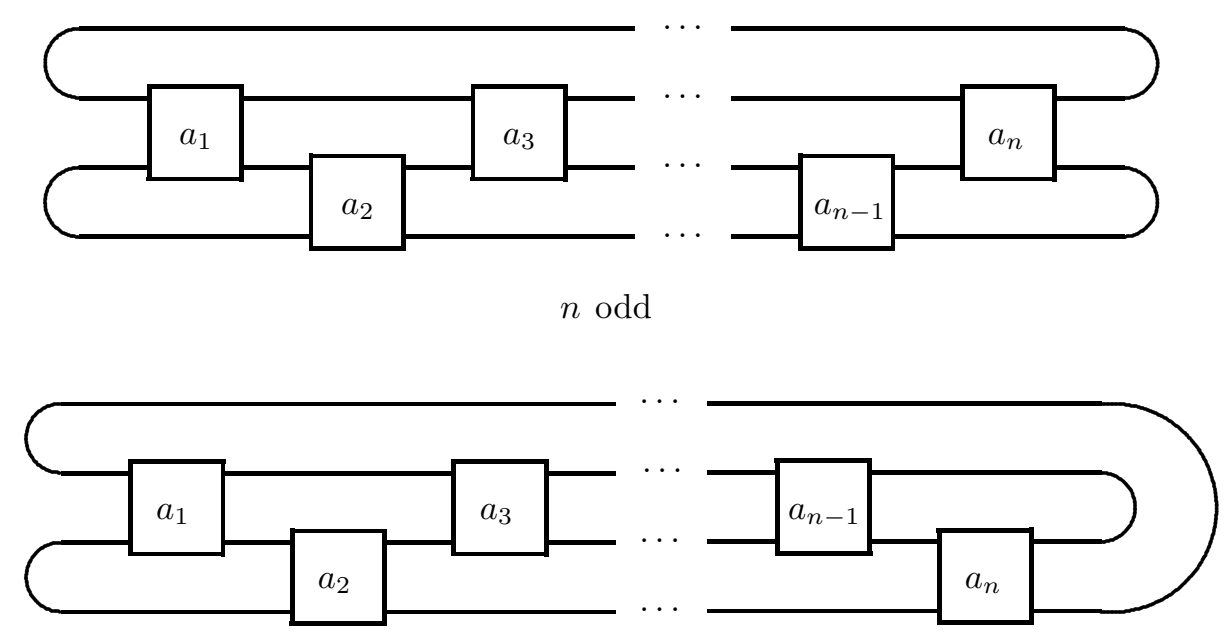

$n$ even

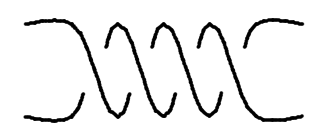

positive twists

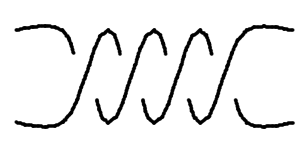

negative twists

Figure 1: The rational-form diagram $T\left(a_{1}, \ldots, a_{n}\right)$. Each box contains the specified number of half-twists; positive and negative twists are shown. 


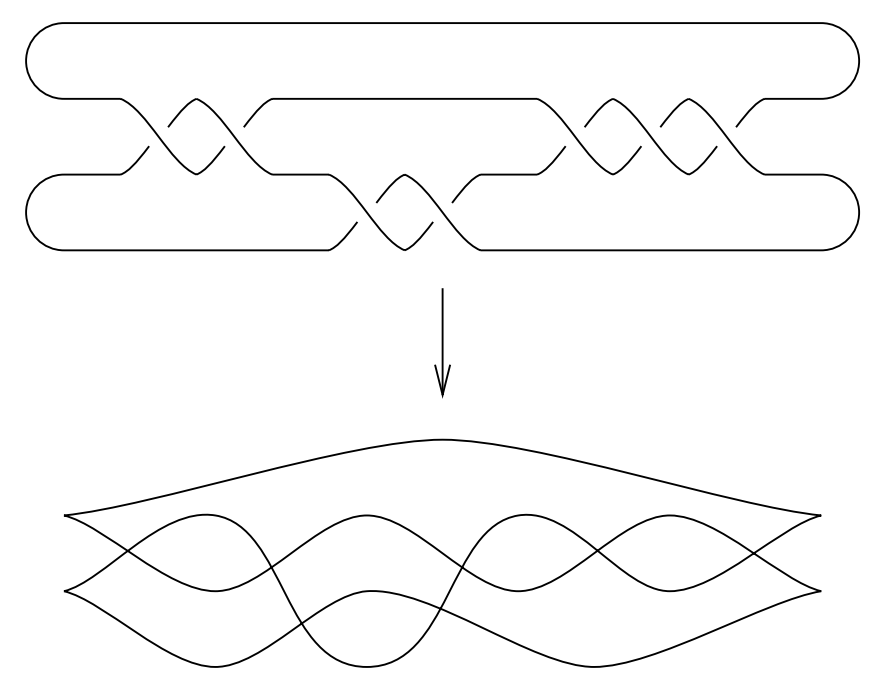

Figure 2: The correspondence between a diagram in Legendrian rational form (in this case, $T(2,2,3)$, or $\left.5_{2}\right)$ and the front of a Legendrian link of the same ambient type.

To each $T\left(a_{1}, \ldots, a_{n}\right)$, we may associate a rational number (or $\left.\infty\right)$, the continued fraction

$$
\left[a_{1}, \ldots, a_{n}\right]=a_{1}+\frac{1}{-a_{2}}+\frac{1}{a_{3}}+\frac{1}{-a_{4}}+\cdots+\frac{1}{(-1)^{n-1} a_{n}} .
$$

Note that our convention is the opposite of the convention in [8], and differs by alternating signs from the standard convention from, e.g., [2]. The classification of 2 -bridge links further states that if $1 /\left[a_{1}, \ldots, a_{n}\right]-1 /\left[b_{1}, \ldots, b_{n}\right] \in \mathbb{Z}$, then $T\left(a_{1}, \ldots, a_{n}\right)$ and $T\left(b_{1}, \ldots, b_{n}\right)$ are ambient isotopic. (The criterion stating precisely when two such links are isotopic is only slightly more complicated, but will not concern us here.)

Now define $T\left(a_{1}, \ldots, a_{n}\right)$ to be in Legendrian rational form if $a_{i} \geq 2$ for all $i$. Although $T\left(a_{1}, \ldots, a_{n}\right)$ corresponds to a Legendrian link whenever $a_{i} \geq 1$ for all $i$, it is crucial to the proof of Lemma 4 below that $a_{i} \geq 2$ for $2 \leq$ $i \leq n-1$. Indeed, if one of these $a_{i}$ is 1 , then it is straightforward to see, by drawing the front, that the resulting Legendrian link does not maximize Thurston-Bennequin number.

Any link diagram in Legendrian rational form is easily converted into the front (i.e., projection to the $x z$ plane) of a Legendrian link by replacing the four vertical tangencies by cusps; see Figure 2. Since the crossings in a front are resolved locally so that the strand with more negative slope always lies over 
the strand with more positive slope, a link diagram in Legendrian rational form is ambient isotopic to the corresponding front. (This observation explains our choice of convention for positive versus negative twists.)

Lemma 3 Any 2-bridge link can be expressed as a diagram in Legendrian rational form.

Proof Let $K$ be a 2-bridge link; let $T\left(a_{1}, \ldots, a_{n}\right)$ be a rational-form diagram for $K$, and write $\left[a_{1}, \ldots, a_{n}\right]=p / q$ for $p, q \in \mathbb{Z}$. The classification of 2 -bridge links implies that $K$ is isotopic to any rational-form diagram associated to the fraction $r=p /\left(q-\left\lfloor\frac{q}{p}\right\rfloor p\right)>1$. (If $q / p$ is an integer, then it is easy to see that $K$ is the trivial knot, which is not 2 -bridge.)

Define a sequence $x_{1}, x_{2}, \ldots$ of rational numbers by $x_{1}=r, x_{i+1}=1 /\left(\left\lceil x_{i}\right\rceil-\right.$ $\left.x_{i}\right)$. This sequence terminates at, say, $x_{m}$, where $x_{m}$ is an integer. Write $b_{i}=\left\lceil x_{i}\right\rceil$. It is easy to see that $b_{i} \geq 2$ for all $i$, and that $r=\left[b_{1}, \ldots, b_{m}\right]$. Then $K$ is isotopic to $T\left(b_{1}, \ldots, b_{m}\right)$, which is in Legendrian rational form.

Consider a link diagram $T=T\left(a_{1}, \ldots, a_{n}\right)$ in Legendrian rational form, and let $K$ be the (Legendrian link given by the) front obtained from $T$. We claim that the Thurston-Bennequin number of $K$ agrees precisely with the Kauffman bound. Recall that the Kauffman polynomial $F_{T}(a, x)$ of $T$ is $a^{w(T)}$ times the unoriented Kauffman polynomial (or L-polynomial) $L_{T}(a, x)$, where $w(T)$ is the writhe of the diagram $T$. (Here we use Kauffman's original notation [7], except with $a$ replaced by $1 / a$.)

We will need a matrix formula for $L_{T}(a, x)$ due to [8]. Write

$$
M=\left(\begin{array}{ccc}
x & -1 & x \\
1 & 0 & 0 \\
0 & 0 & 1 / a
\end{array}\right), \quad S=\left(\begin{array}{ccc}
0 & 1 & 0 \\
0 & 0 & 1 \\
1 / a & 0 & 0
\end{array}\right), \quad v=\left(\begin{array}{l}
1 \\
0 \\
0
\end{array}\right), \quad w=\left(\begin{array}{c}
a \\
a^{2} \\
\frac{a^{2}+1}{x}-a
\end{array}\right)
$$

then

$$
L_{T\left(a_{1}, \ldots, a_{n}\right)}(a, x)=\frac{1}{a} v^{t} M^{-a_{1}-1} S M^{-a_{2}-1} S \cdots M^{-a_{n}-1} S w,
$$

where $t$ denotes transpose.

Lemma 4 If $a_{1}, a_{n} \geq 1$ and $a_{i} \geq 2$ for $2 \leq i \leq n-1$, then we have $\min -\operatorname{deg}_{a} L_{T\left(a_{1}, \ldots, a_{n}\right)}(a, x)=-1$.

Proof None of $M^{-1}, M^{-1} S$, and $w$ contains negative powers of $a$; the lemma will be proved if we can show that $f(x) \neq 0$, where

$$
f(x)=\left.\left(v^{t} M^{-a_{1}}\left(M^{-1} S\right) M^{-a_{2}}\left(M^{-1} S\right) \cdots M^{-a_{n}}\left(M^{-1} S\right) w\right)\right|_{a=0} .
$$


Define the auxiliary matrices

$$
A=\left.M^{-1}\right|_{a=0}=\left(\begin{array}{ccc}
0 & 1 & 0 \\
-1 & x & 0 \\
0 & 0 & 0
\end{array}\right), \quad B=\left.\left(M^{-2} S M^{-1}\right)\right|_{a=0}=\left(\begin{array}{ccc}
1 & 0 & 0 \\
x & 0 & 0 \\
0 & 0 & 0
\end{array}\right), \quad u=\left(\begin{array}{l}
0 \\
1 \\
0
\end{array}\right) .
$$

Then $\left.(A S w)\right|_{a=0}=\frac{1}{x} A u$ and $B=A u v^{t}$, and so

$$
\begin{aligned}
f(x) & =\left.v^{t} A^{a_{1}-1} B A^{a_{2}-2} B A^{a_{3}-2} B \cdots A^{a_{n-1}-2} B A^{a_{n}-1}(A S w)\right|_{a=0} \\
& =\frac{1}{x}\left(v^{t} A^{a_{1}} u\right)\left(v^{t} A^{a_{2}-1} u\right)\left(v^{t} A^{a_{3}-1} u\right) \cdots\left(v^{t} A^{a_{n-1}-1} u\right)\left(v^{t} A^{a_{n}} u\right) .
\end{aligned}
$$

But if we define a sequence of functions $f_{k}(x)=v^{t} A^{k} u$, then an easy induction yields the recursion $f_{k+2}(x)=x f_{k+1}(x)-f_{k}(x)$ with $f_{1}(x)=1$ and $f_{2}(x)=x$. In particular, for all $k \geq 1, f_{k}(x)$ has degree $k-1$ and is thus nonzero. From the given conditions on $a_{i}$, it follows that $f(x) \neq 0$, as desired.

Proof of Theorem 1 Let $T$ be a Legendrian rational form for a 2-bridge link $K$. The crossings of $T$ are counted, with the same signs, by both the writhe of $T$ and the Thurston-Bennequin number of the Legendrian link $K^{\prime}$ obtained from $T ; t b\left(K^{\prime}\right)$, however, also subtracts half the number of cusps. Hence

$$
\begin{aligned}
t b\left(K^{\prime}\right) & =w(T)-2 \\
& =\left(\min -\operatorname{deg}_{a} F_{T}(a, x)-\min -\operatorname{deg}_{a} L_{T}(a, x)\right)-2 \\
& =\min -\operatorname{deg}_{a} F_{T}(a, x)-1
\end{aligned}
$$

by Lemma 4 . Since $K^{\prime}$ is ambient isotopic to $K$, we conclude that $\overline{t b}(K)$ is at least min- $\operatorname{deg}_{a} F_{T}(a, x)-1$; by the Kauffman bound, equality must hold.

\section{Appendix: Maximal Thurston-Bennequin number for small knots}

The following table gives the maximal Thurston-Bennequin invariant for all prime knots with nine or fewer crossings. We distinguish between mirrors by using the diagrams in [10]: the knots $K$ are the ones drawn in [10], with mirrors $\tilde{K}$. A dagger next to a knot indicates that it is not two-bridge; a double dagger indicates that the knot is amphicheiral (identical to its unoriented mirror). For the interested reader, two-bridge descriptions of the two-bridge knots in the table can be deduced from the tables in [2].

The boldfaced numbers indicate the knots for which the Kauffman bound is not sharp (for 819 ), or probably not sharp (for $9_{42}$ ). As mentioned in the Introduction, it is believed that $\overline{t b}=-5$ for the mirror $9_{42}$ knot; the best known bound, however, is the Kauffman bound $\overline{t b} \leq-3$. 


\begin{tabular}{|c|c|c|c|c|c|c|c|c|}
\hline$K$ & $\overline{t b}(K)$ & $\overline{t b}(\tilde{K})$ & $K$ & $\overline{t b}(K)$ & $\overline{t b}(\tilde{K})$ & $K$ & $\overline{t b}(K)$ & $\overline{t b}(\tilde{K})$ \\
\hline$\overline{0_{1}}$ & $\overline{-1}$ & $\mp$ & $\overline{8}_{15^{\dagger}}^{\dagger}$ & $\overline{-13}$ & $\overline{3}$ & $\overline{9_{22}{ }^{\dagger}}$ & $\overline{-3-3}$ & -8 \\
\hline $3_{1}$ & -6 & 1 & $8_{16}^{\dagger}$ & -8 & -2 & $9_{23}$ & -14 & 3 \\
\hline $4_{1}$ & -3 & $\ddagger$ & $8_{17}^{\dagger}$ & -5 & $\ddagger$ & $9_{24}^{\dagger}$ & -6 & -5 \\
\hline $5_{1}$ & -10 & 3 & $8_{18}^{\dagger}$ & -5 & $\ddagger$ & $9_{25}^{\dagger}$ & -10 & -1 \\
\hline $5_{2}$ & -8 & 1 & $8_{19}^{\dagger}$ & 5 & -12 & $9_{26}$ & -2 & -9 \\
\hline $6_{1}$ & -5 & -3 & $8_{20}^{\dagger}$ & -6 & -2 & $9_{27}$ & -6 & -5 \\
\hline $6_{2}$ & -7 & -1 & $8_{21}^{\dagger}$ & -9 & 1 & $9_{28}^{\dagger}$ & -9 & -2 \\
\hline $6_{3}$ & -4 & $\ddagger$ & $9_{1}$ & -18 & 7 & $9_{29}^{\dagger}$ & -8 & -3 \\
\hline $7_{1}$ & -14 & 5 & $9_{2}$ & -12 & 1 & $9_{30}^{\dagger}$ & -6 & -5 \\
\hline $7_{2}$ & -10 & 1 & $9_{3}$ & 5 & -16 & $9_{31}$ & -9 & -2 \\
\hline $7_{3}$ & 3 & -12 & $9_{4}$ & -14 & 3 & $9_{32}^{\dagger}$ & -2 & -9 \\
\hline $7_{4}$ & 1 & -10 & $9_{5}$ & 1 & -12 & $9_{33}^{\dagger}$ & -6 & -5 \\
\hline $7_{5}$ & -12 & 3 & $9_{6}$ & -16 & 5 & $9_{34}^{\dagger}$ & -6 & -5 \\
\hline $7_{6}$ & -8 & -1 & $9_{7}$ & -14 & 3 & $9_{35}^{\dagger}$ & -12 & 1 \\
\hline $7_{7}$ & -4 & -5 & $9_{8}$ & -8 & -3 & $9_{36}^{\dagger}$ & 1 & -12 \\
\hline 81 & -7 & -3 & $9_{9}$ & -16 & 5 & $9_{37}^{\dagger}$ & -6 & -5 \\
\hline $8_{2}$ & -11 & 1 & $9_{10}$ & 3 & -14 & $9_{38}^{\dagger}$ & -14 & 3 \\
\hline $8_{3}$ & -5 & $\ddagger$ & $9_{11}$ & 1 & -12 & $9_{39}^{\dagger}$ & -1 & -10 \\
\hline $8_{4}$ & -7 & -3 & $9_{12}$ & -10 & -1 & $9_{40}^{\dagger}$ & -9 & -2 \\
\hline $8_{5}^{\dagger}$ & 1 & -11 & $9_{13}$ & 3 & -14 & $9_{41}^{\dagger}$ & -7 & -4 \\
\hline $8_{6}$ & -9 & -1 & $9_{14}$ & -4 & -7 & $9_{42}^{\dagger}$ & -3 & $-5(?)$ \\
\hline 87 & -2 & -8 & $9_{15}$ & -10 & -1 & $9_{43}^{\dagger}$ & 1 & -10 \\
\hline 88 & -4 & -6 & $9_{16}^{\dagger}$ & 5 & -16 & $9_{44}^{\dagger}$ & -6 & -3 \\
\hline 89 & -5 & $\ddagger$ & $9_{17}$ & -8 & -3 & $9_{45^{\dagger}}^{\dagger}$ & -10 & 1 \\
\hline $8_{10}^{\dagger}$ & -2 & -8 & $9_{18}$ & -14 & 3 & $9_{46}^{\dagger}$ & -7 & -1 \\
\hline $8_{11}$ & -9 & -1 & $9_{19}$ & -6 & -5 & $9_{47}^{\dagger}$ & -2 & -7 \\
\hline $8_{12}$ & -5 & $\ddagger$ & $9_{20}$ & -12 & 1 & $9_{48}^{\dagger}$ & -1 & -8 \\
\hline $8_{13}$ & -4 & -6 & $9_{21}$ & -1 & -10 & $9_{49}^{\dagger}$ & 3 & -12 \\
\hline $8_{14}$ & -9 & -1 & & & & & & \\
\hline
\end{tabular}




\section{References}

[1] D Bennequin, Entrelacements et équations de Pfaff, Astérisque 107-108 (1983) $87-161$

[2] G Burde, H Zieschang, Knots, Walter de Gruyter (1985)

[3] J Epstein, On the invariants and isotopies of Legendrian and transverse knots, $\mathrm{PhD}$ thesis, U C Davis, 1997

[4] J Etnyre, K Honda, Knots and contact geometry, preprint, 2000, arXiv: math.GT/0006112

[5] E Ferrand, On Legendrian knots and polynomial invariants, preprint, 2000, arXiv:math.GT/0002250

[6] D Fuchs, S Tabachnikov, Invariants of Legendrian and transverse knots in the standard contact space, Topology 36 (1997) 1025-1054

[7] L Kauffman, On Knots, Princeton University Press (1987)

[8] W B R Lickorish, Linear skein theory and link polynomials, Topology Appl. 27 (1987) 265-274

[9] L Ng, Invariants of Legendrian links, PhD thesis, MIT, 2001, available at http://www-math.mit.edu/ lenny/

[10] D Rolfsen, Knots and Links, Publish or Perish (1990)

[11] L Rudolph, A congruence between link polynomials, Math. Proc. Camb. Phil. Soc. 107 (1990) 319-327

[12] H Schubert, Knoten mit zwei Brücken, Math. Zeit. 65 (1956) 133-170

[13] T Tanaka, Maximal Bennequin numbers and Kauffman polynomials of positive links, Proc. Amer. Math. Soc. 127 (1999) 3427-3432

[14] N Yufa, Thurston-Bennequin invariant of Legendrian knots, senior thesis, MIT, 2001

Department of Mathematics, Massachusetts Institute of Technology,

77 Massachusetts Avenue, Cambridge, MA 02139, USA

Email: ng@alum.mit.edu

URL: http://www-math.mit.edu/ lenny/

Received: 24 May 2001 Revised: 26 July 2001 